Research Article

\title{
Image Recognition of Pledges of Capital Stock in Small- and Medium-Sized Enterprises Based on Partial Differential Equations
}

\author{
Dehui Zhou \\ School of Finance, Xi'an Eurasia University, Xi'an, Shaanxi 710065, China \\ Correspondence should be addressed to Dehui Zhou; zhoudehui@eurasia.edu
}

Received 17 September 2021; Accepted 16 October 2021; Published 2 November 2021

Academic Editor: Miaochao Chen

Copyright ( 2021 Dehui Zhou. This is an open access article distributed under the Creative Commons Attribution License, which permits unrestricted use, distribution, and reproduction in any medium, provided the original work is properly cited.

\begin{abstract}
Image recognition is one of the core research directions in the field of computer vision research, which can be divided into general image recognition and fine-grained image recognition. General image recognition refers to the recognition of different types of objects; fine-grained image recognition refers to the recognition of different subclasses in the same broad class of objects, such as SME financing inventory pledge image recognition. In this paper, we propose a partial differential equation-based image recognition method for SME financing inventory pledges and conduct detailed analysis and experiments. Compared with general images, partial differential equation-based SME financing inventory pledges image recognition is difficult to recognize due to data characteristics such as small differences in features between classes, large differences in features within classes, and a small percentage of targets in the image. To address the problem that existing methods ignore the role of shallow features on fine-grained image recognition, this paper proposes a fine-grained image recognition method based on partial differential equations. By analyzing the important role of shallow features for fine-grained image recognition, a feature fusion method with adaptive weights is proposed. Using this method to fuse shallow and high-level semantic features for recognition, the role of shallow features in fine-grained image recognition is fully exploited. In addition, the proposed method does not change the order of magnitude of the model parameters and is highly transferable. The relevant experimental results verify the effectiveness of the proposed method.
\end{abstract}

\section{Introduction}

The invention of electronic computers during the third industrial revolution led to the information age. With the development of the information age, the future society will have $90 \%$ of its information traffic from visual data, i.e., image and video data. Currently, the global Internet alone can generate petabytes of data of order of magnitude per day in terms of visual information volume [1]. This data contains a wealth of information that is useful not only for scientific research but also for life. Effective use of this visual information is an important research component for industry and academia. Initially, humans relied only on themselves to process this data, but the rate at which humans could process data on their own was much slower than the rate at which the Internet could generate data. With the rapid development of computer technology, computers have long been able to process data much faster than humans. Therefore, human beings want to process these visual data with the help of computers, thus forming the field of computer vision research. Computer vision has a wide range of application prospects in medical, transportation, security, science, agriculture, military, and other fields, mainly containing image recognition, target detection, target tracking, and image generation, and other research subdirections, of which image recognition is the basis for other tasks.

Before the actual processing of the image, image recognition processing is required. The presence of noise can affect the subsequent image processing results, such as the inability to obtain accurate edge information in image edge extraction and reduced recognition capability of the model in image recognition. 
Therefore, image recognition is highly researchable. Noise in images originates from processes such as image generation and transmission, which manifests itself in images with a machine with discrete pixel points, and the presence of noise can degrade the image quality and affect people's use. Image recognition can bring people sharper, higher-quality images [2].

Image coloring is yet another task in image processing. Image coloring, as the name implies, means adding color to an image, and the technique. Not only can you colorize grayscale images, but you can also color transform existing color images. This can be achieved through the processing of the image is colored to increase the visual effect of the image and to facilitate in-depth research and analysis of the image. Image coloring has a wide range of applications in life [3], such as ancient paintings that can have varying degrees of deterioration due to time; image coloring can be used for the ancient paintings are restored to increase their ornamental and artistic value; for black-and-white photos and black-and-white films, the image coloring can increase strong image authenticity; also image coloring of medical grayscale images, easy for medical personnel to observe and diagnose, etc.

\section{Related Work}

The research on image recognition has developed to date with fruitful results. It includes methods based on the transform domain and spatial domain, vector-based diffusion, and tensor-based diffusion. In recent years, with the development of deep learning, image recognition has also shifted from traditional methods to deep learning, neural networkbased methods. Today, there are still many scholars working on the image recognition problem. Recognition methods based on the image transform domain include wavelet transform and Fourier transform, and recognition methods based on image space domain methods such as Wiener filtering and adaptive median filtering, which can achieve recognition but at the same time can damage the image in some edge information cannot meet the needs of people. The literature has creatively used partial differential equation (PDE) to study image smoothing and enhancement, opening up PDE-based image processing [4].

After that, the literature provided a theoretical basis for the use of PDEs for image recognition. By the 1990s, the partial differential equation-based image processing method gradually became one of the popular methods in the field of image processing. At present, the method has achieved fruitful results. The earliest partial differential equation applied to image recognition is the heat conduction equation, which corrupts the image edges to some extent while recognizing them. Based on the heat conduction equation, an anisotropic diffusion model (i.e., P-M model) was proposed in the literature, which can preserve image boundaries well, but the uniqueness of the solution cannot be guaranteed as the model is pathological. The literature proposes the more stable regularized P-M model, which is an optimization of the P-M model. The curvature change-based model proposed in the literature also achieves some identification results. The P-M model and its improved model are both second-order PDE models, which produce "step effect" in recognition, while the higher-order PDE model proposed by scholars can effectively solve the problem [5]. The fourth-order PDE model (Y-K model) is proposed in the literature to eliminate the "step effect," but the speckle phenomenon also appears. The diffusion coefficient of the Y-K model is modified, and the recognition effect is improved compared with the Y-K model. A two-step algorithm is proposed to remove the pretzel noise. The literature proposes a recognition method for Poisson noise by maximum a posteriori estimation (MAP). In addition, many scholars have proposed fourth-order PDE models, fractional-order PDE models, and PDE-based "hybrid" recognition models, which have achieved some recognition results. A recognition model based on total variation (TV) is proposed in the literature, which preserves the image edge information while recognizing. According to this idea, various adaptive TV models have been proposed by scholars. The literature has proposed a variational model that can effectively remove the step effect by using higher-order derivatives to smoothly approximate the function. Recognition models based on full variance have been extensively studied in the literature, and all achieve desirable recognition results in terms of recognition.

The color transfer-based coloring method is to perform the color transfer in two similar images, the specific process is first input A grayscale image, then a color image similar to the grayscale image is input, and the grayscale image is realized through computer technology with matching the transfer of pixels between color images leads to the colorization of grayscale images. The literature implemented color transfer through block similarity. Subsequently, the literature people implemented image coloring by combining the Bayesian image mapping algorithm and image transfer. Since each pixel point is treated individually, these methods suffer from the problem of spatial consistency. To overcome this problem, scholars have applied image segmentation to image coloring. In addition to this, other color transfer-based coloring methods exist. The literature considers that the image coloring problem can be treated as an image restoration problem. The authors in $\mathrm{YCbCr}$ color space propose a full variational model to obtain the chromaticity information of the image by solving this optimization problem and then combine it with the luminance information of the image to obtain the colorized image. However, due to the nonconvexity of this model, the coloring results will be affected by the initial contour lines. Later, the regenerating kernel Hilbert space (RKHS (reproducing kernel Hilbert spaces)) was used for image coloring, and the coloring performance was improved, but the model is still nonconvex. In addition, many other scholars have studied the full variational model. In all of these methods mentioned above, the gradient information of the image is used to control the color diffusion, but the color diffusion cannot be achieved correctly due to the influence of pseudoedges in the image. To address the effect of pseudoedges in images, the literature proposes a coupled full-variance model that performs fast coloring in smooth regions and stops color diffusion at edges. Since the Jin model is convex and insensitive to initial values, it 
has good robustness. In addition, the literature proposes a nonlocal estimation-based image coloring method by combining the texture features of the image. In paper [5], the authors use higher-order regularization for image coloring, which can suppress the color diffusion at the edges of the image. The literature proposes a method for selecting seed pixels that improve the effectiveness of manually adding colors. The literature proposes two alternate algorithms to solve the nonconvex full variational model, and the results also outperform the existing algorithms [6].

\section{Application of Partial Differential Equation- Based Image Recognition in SME}

Private SMEs are an important link to ensure the employment of workers and the main nature of the market and also contribute to the development of the local economy and the stability of the regional industrial chain supply chain. The theory of partial differential equations is applied in many practical problems, and people analyze the image and then build the PDE model that best fits the bar so that the image can be processed better. With the improvement and development of partial differential equation theory, PDE-based image processing methods have received more and more attention in recent years. This section focuses on the application of PDE in remote sensing image noise reduction, including the mathematical theory of partial differential equation and the principle of classical PDE recognition model, etc., and the experimental analysis compares the recognition effect of related algorithms.

3.1. The Underlying Logic of Partial Differential Equations. A partial differential equation is defined in the field of mathematics as an equation that contains the partial derivatives of an unknown function [7]. The equation is inseparably related to many practical problems not only in the field of physics but also in the field of mathematics when it is applied in practice. The equation abstracts all these practical problems and obtains a mathematical model, and the desired results can be achieved by using different models depending on the purpose of the treatment. Because the problems solved by this equation are mainly from physics, it is also called a mathematical physics equation. Many branches of physics and mathematics use the theory of partial differential equations due to the growing relationship between the various disciplines. Its formulation theory is

$$
T=\frac{d y}{d x} \cdot \frac{\partial^{2} \Omega}{\partial v^{2}}
$$

In the 1980s, the concept of scale space was introduced to image processing to decompose images at multiple scales, and this was the earliest study of partial differential equation theory in the field of image processing. In image processing, the basic principle of partial differential equations is to meet the needs of practical image processing, so the most qualified mathematical model should be selected to create the abstract model, and the solution after solving the equation is the result we hope to get; PDE-based image processing algo- rithms have the following advantages compared with traditional methods [8]. The first formula of the article is the core formula of the article, which ensures that the algorithm can perform the basic function of image recognition, which is to use the method based on the partial differential equation to recognize the image.

(1) The PDE is inseparable from the real problem, and a variety of geometric features such as the gradient of the image as well as the orientation information can be selected as processing elements of the model when performing image processing. The theory of its formulation is

$$
T=\frac{(\Delta y / \Delta x)}{\left(\partial^{2} \Omega / \partial v^{2}\right)}
$$

(2) The PDE algorithm is based on the traditional discrete filtering method for generalization and continuity analysis, and the algorithm can use a continuous model when analyzing images. The theory of its formulation is

$$
A=\frac{1}{n} \sum_{i=1}^{n}\left(X_{i}-\bar{X}\right)^{2}
$$

(3) Partial differential equations, due to their own very strong mathematical theoretical foundation, can be combined with many already well-established numerical computational methods in the field of mathematics, such as simulated degeneracy and genetic algorithms. The theory of its formulation is

$$
B=\lim _{n \longrightarrow \infty} \sum_{i=1}^{n} X_{i}^{2} \cdot \lim _{n \longrightarrow \infty} \sum_{i=1}^{n} X_{i} Y_{i}
$$

At present, partial differential equation processing methods are widely used in image processing-related fields such as image noise reduction, edge detection, and image segmentation. In recent years, image processing techniques based on partial differential equation methods are a key research branch in the field of image processing and have attracted the attention of people in related fields. Problems such as image smoothing and image enhancement are studied. At the same time, the literature has made a breakthrough in the exploration of both scale space and image structure in the field of image processing [9]. Later, the literature found that the parabolic partial differential equations in the scale space can be established using the thermal diffusion equation, while the evolution equations to satisfy the maximum principle can be done by defining a scale space. 
The image recognition process based on the partial microequation mainly combined the feature selection problem with a genetic algorithm, simulated annealing algorithm, particle swarm optimization algorithm, random forest, or a random resampling process in the calculation of this kind of method and use probabilistic reasoning and sampling process. As the basis of the algorithm, based on the effectiveness of classification estimation, a certain weight is assigned to each feature during the operation of the algorithm, and then, the importance of the feature is evaluated according to a given or adaptive threshold. Other branches of higher mathematics and image processing, such as mathematical morphology, image horizontals, and image shapes, provide useful theoretical justifications for the development of this new method. Among them, image filtering and image segmentation in image processing became the immediate conditions for the eventual formation of this discipline. The theory of its formulation is

$$
B=\frac{\sum_{i=1}^{n} X_{i}^{2}}{\sum_{i=1}^{n} X_{i} Y_{i}} .
$$

The theory of partial differential equation recognition was first introduced by Gaussian filtering. Because the Gaussian filtering linear diffusion uses the same diffusion strength and method at the flat areas and edges of the image, so although the method filters the noise well, the detailed texture and edge features of the image are significantly damaged and the quality of the filtered image suffers severely. PDE-based image processing methods cover almost the entire field of image processing and since the PDE based [10].

The image processing method is a low-level image processing, so many other image processes such as feature extraction, image segmentation, and image restoration can apply its results as intermediate results. Partial differential equations were first applied mainly in the field of physics to help people solve problems in physics, and as people studied partial differential equations more, it was applied to many branches of physics and mathematics. The simultaneous existence of the variables in an equation and their derivatives of all orders in the same equation is the most important feature of partial differential equations. A normally differential equation is one in which the unknown function in the equation contains only one independent variable, whereas a partial differential equation is one in which the unknown function in the equation is related to more than one variable and there are derivatives of the unknown function concerning more than one variable in the equation at the same time.

There are three types of partial differential equations that are often used in practical applications, mainly the Laplace equation, the heat conduction equation, and the fluctuation equation. They are considered to be the most typical partial differential equations and are the first partial differential equations used in physics and mathematics. Since the solutions of these three equations can solve many important physical problems, it has a very important and wide range of applications. Here are the specific expressions for these three equations.

(1) Laplace's equation

$$
M=\frac{\sum_{i=1}^{n} X_{i}^{2}}{(x-\mu) / \sigma}
$$

(2) Heat conduction equation

$$
\frac{x-\mu}{\sigma}=\sum_{i=1}^{n} X_{i} Y_{i}+\sum_{i=1}^{n} X_{i}^{2}
$$

(3) Wave equation

$$
N=\sum_{i=1}^{n} X_{i} Y_{i}+\sum_{i=1}^{n} X_{i}^{2}+\sum_{i=1}^{n} X_{i}
$$

3.2. Partial Differential Equation-Based Asset Inventory Charge Image Recognition. A brief description of the product image samples used in the image recognition experiments will be given. Since there is no supermarket inventory database available directly on the Internet, the shelf inventory database used in this paper for the study of supermarket shelf product images is derived from actual images of supermarket shelf inventory, since different types of products have different shapes in themselves and there are some differences in the distance and angle at which the images are taken. Therefore, the dimensions of each product sample obtained by image segmentation may vary. Therefore, to reduce the error of image recognition, the original images obtained need to be standardized, so that the images of the inventory library have the same size through processing, which is more conducive to image recognition, and different labels are labeled for different types of inventory to complete the design of the shelf inventory image library [11]. Before using the algorithm used in this article, the picture must first be refined to ensure that it can be recognized. For the refinement process, the clarity of the image must be guaranteed first, and then, the details of the image must be controlled.

3.2.1. Image Recognition Steps. To identify the shelf inventory more effectively, the inventory image recognition scheme designed in this paper includes image acquisition, image preprocessing, feature extraction, and classification recognition into four parts:

(1) Image acquisition: the images used in this experiment were collected from the actual shelf images of supermarkets. Beverages, food items, and daily necessities were selected from the experimental 
sample images as part of the product category for the experiment

(2) Image preprocessing: manual segmentation of each type of inventory in the collected shelf inventory images is performed to complete the process of building the experimental sample library. First, the product categories for the experiments are selected and the image samples of each category are segmented from different angles and finally scaled to a uniform size. The principle of the formula is

$$
H=\sum_{i=1}^{n} X_{i} Y_{i}+\sum_{i=1}^{n} X_{i}^{2}+\frac{x-\mu}{\sigma}
$$

(3) Image feature extraction: by using principal component analysis and linear discriminant analysis, features of shelf product images are extracted and the obtained features are used in the next step of image recognition

(4) Classification recognition [12]: the classifier is trained using multiple classifiers by using different classification decision criteria while using samples of the inventory images for the classifier to be relevant after the classifier training is completed, the extracted features of the inventory to be recognized are input for image recognition and the classification results of the samples to be recognized are derived. The block diagram of the inventory recognition process is shown in Figure 1

3.2.2. SME Capital Inventory Charge Image Feature Extraction. Image recognition can essentially be seen as a classification process. To be able to accurately identify the category to which an image belongs, it needs to be distinguished from different categories of images. This requires that the selected image feature not only needs to have good image description properties but also that the feature can distinguish images from different classes. In this section, two image feature dimensionality reduction methods, PCA and LDA, will be used to reduce the dimensionality of the image features to be recognized, respectively; for the sake of operational simplicity, the color image is usually grayed out, and the recognition difficulty of the transformed image is greatly reduced. The operation of converting a color image to a grayscale image is called grayscale processing of the image. Each pixel in a color image is determined by the three color components of R, G, and B, and each component has 255 cases. Thus, a pixel is capable of having a range of more than 16 million $(255 * 255 * 255)$ color transformations [13]. A grayscale image is a special color image with the same R, G, and B components. A pixel has a range of 255 . Therefore, when performing the processing of digital images, image grayscale processing is performed first to reduce the computational effort for subsequent image recognition. The transformed grayscale image has the same image description as the color image and can represent the local and overall chromaticity and luminance distribution characteristics of the entire image. The grayscale processing of the image can be implemented in the following manner.

\section{(1) Maximum value method}

The color feature with the highest brightness among the three color components in the color image is taken as the grayscale value of the grayscale map. The principle of the formula is as follows:

$$
M=\sum_{i=1}^{n}\left(X_{i}-\bar{X}\right)^{2}+\frac{1}{n}\left(\frac{x-\mu}{\sigma}\right)
$$

\section{(2) Average method}

The sum of the luminance of the three color components of the color image is averaged, and the average value obtained is the gray value. The principle of the formula is as follows:

$$
M=\sum_{i=1}^{n}\left(X_{i}-\bar{X}\right)^{2}+\sum_{i=1}^{n} X_{i} Y_{i}-\frac{x-\mu}{\sigma}
$$

\section{(3) Weighted average method}

The three color components of R, G, and B are weighted and averaged using different weights according to the importance of color recognition and other related indicators. Because the human eye is relatively sensitive to green, while having the lowest sensitivity to blue, the three color components are weighted and evaluated separately according to the following weights based on the inductive analysis of several trials, which can ensure that the converted grayscale image is reasonable. The principle of the formula is as follows:

$$
M=\frac{1}{n} \sum_{i=1}^{n} X_{i} Y_{i}+\frac{1}{n} \sum_{i=1}^{n} X_{i}
$$

The three methods of performing grayscale all have their conditions of applicability; for the identification of stock images, after several experiments for comparison and validation, it can be obtained that the grayscale operation of the original image by the weighted average valuation method is more effective for grayscale images, color images, and grayscale. The principal component analysis (PCA) method, by using a set of low-dimensional data can effectively retain most of the information in the original data. Therefore, experimenting with a two-dimensional matrix consisting of product sample images [14], feature extraction using the PCA method not only reduces the computational effort significantly but also represents the original product image as accurately as possible and preserves the overall structural information of the image to the maximum extent possible. 


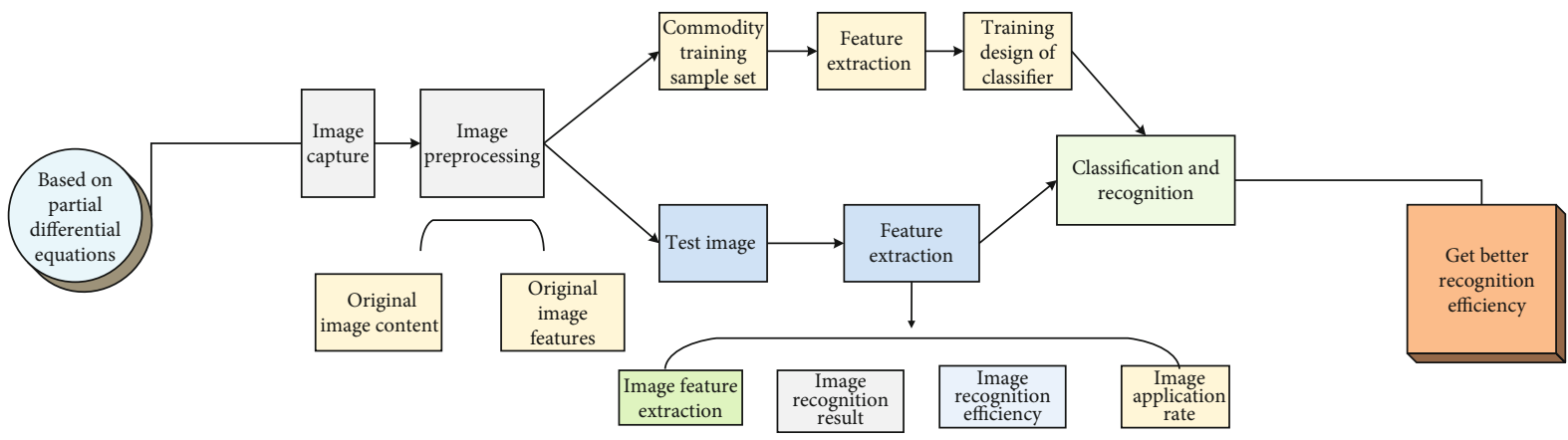

FIgURE 1: Inventory identification process.

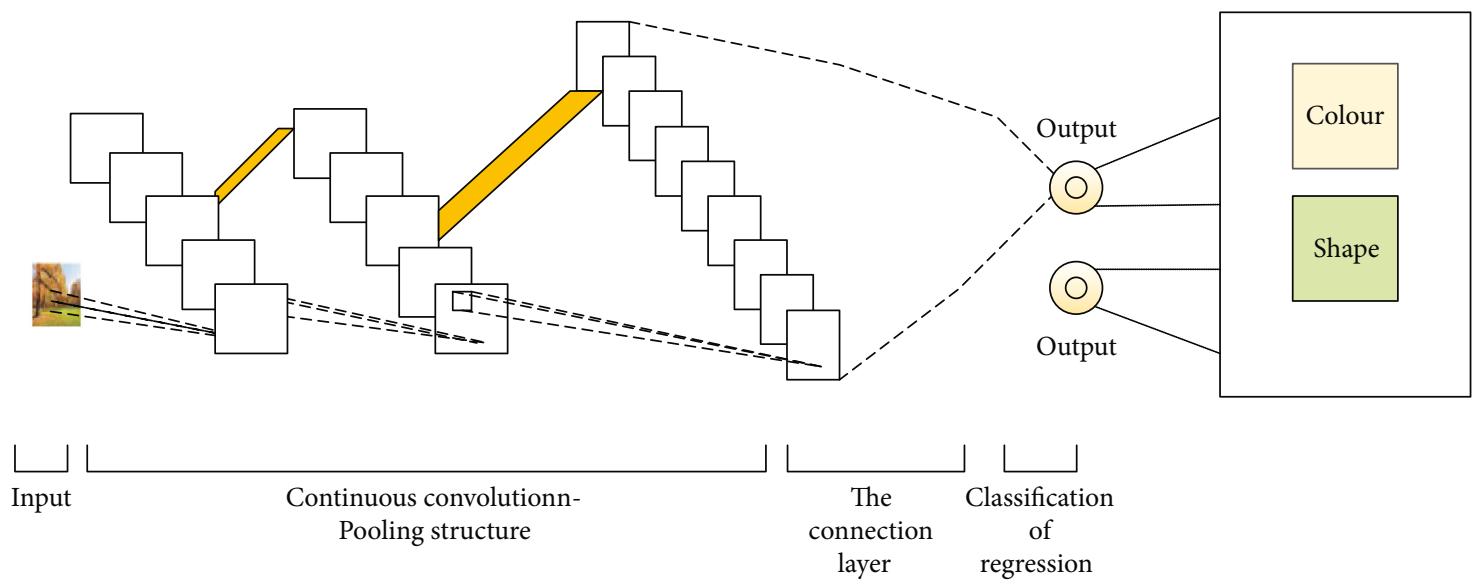

FIGURE 2: Image recognition structure.

TABle 1: Average distance method and error correction SVM identification results based on PCA features with different feature dimensions (in \%).

\begin{tabular}{lccc}
\hline Recognition methods & Precision & Quantity & Efficient \\
\hline Partial differential equation & 90 & 95 & 98 \\
Error correction SVM & 80 & 85 & 88 \\
Average distance method & 72 & 81 & 83 \\
\hline
\end{tabular}

The process of inventory image feature extraction using principal component analysis is to perform a K-L transformation on the entire covariance matrix of the training sample set to obtain a set of feature vectors, which are then used to form a feature subspace onto which each training and test sample can be projected, and a set of coordinate coefficients can be obtained, indicating the location of the image to be tested in the subspace. Then, by comparing the coordinate values of the test samples with the training samples, the final result can be obtained by classifier recognition. The resulting process is shown in Figure 2 [15].

(1) The captured inventory images are the input. Then, a portion of the images of each product in the product image library is selected as the training sample set to obtain the vector set, which is represented by the formula principle:

$$
M=\lim _{x \longrightarrow \infty} \sum_{i=1}^{n} X_{i}^{2}
$$

(2) Calculate the overall dispersion (covariance) matrix. The following functional expression can be obtained:

$$
A=\lim _{x \longrightarrow \infty} \sum_{i=1}^{n} X_{i}^{2}+\lim _{x \longrightarrow \infty} \sum_{i=1}^{n} X_{i} Y_{i}
$$

(3) Solving for the eigenvalues and eigenvectors of the scatter matrix. From the above equation, we can see that the scatter matrix is an $\mathrm{MN} * \mathrm{MN}$ dimensional matrix, but the value of $\mathrm{MN}$ is very large, if the eigenvalues and eigenvectors of this matrix are solved directly, the computation is very large and generally takes a lot of time, to save time and improve the efficiency of image recognition; the method of singular value decomposition (SVD) is used for indirect solving. The basic principle of singular value decomposition (SVD) is 


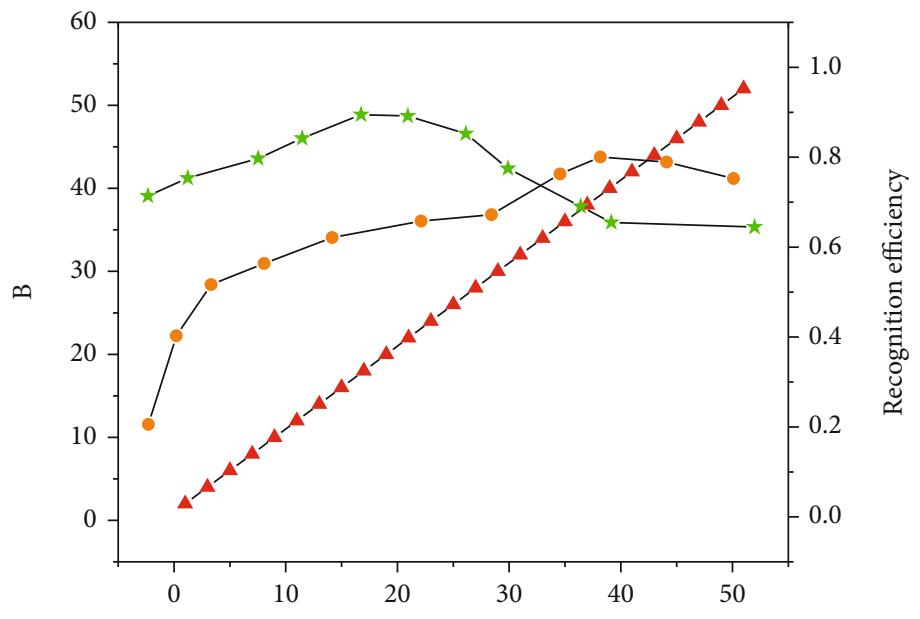

Number of items identified by different algorithms

$$
\begin{aligned}
& \text { - } \Delta \text { - SVM } \\
& \text {-๑- Partial differential equation } \\
& \text { - - Other algorithms }
\end{aligned}
$$

FIgURE 3: Average distance method based on PCA features and the result of corrected image recognition.

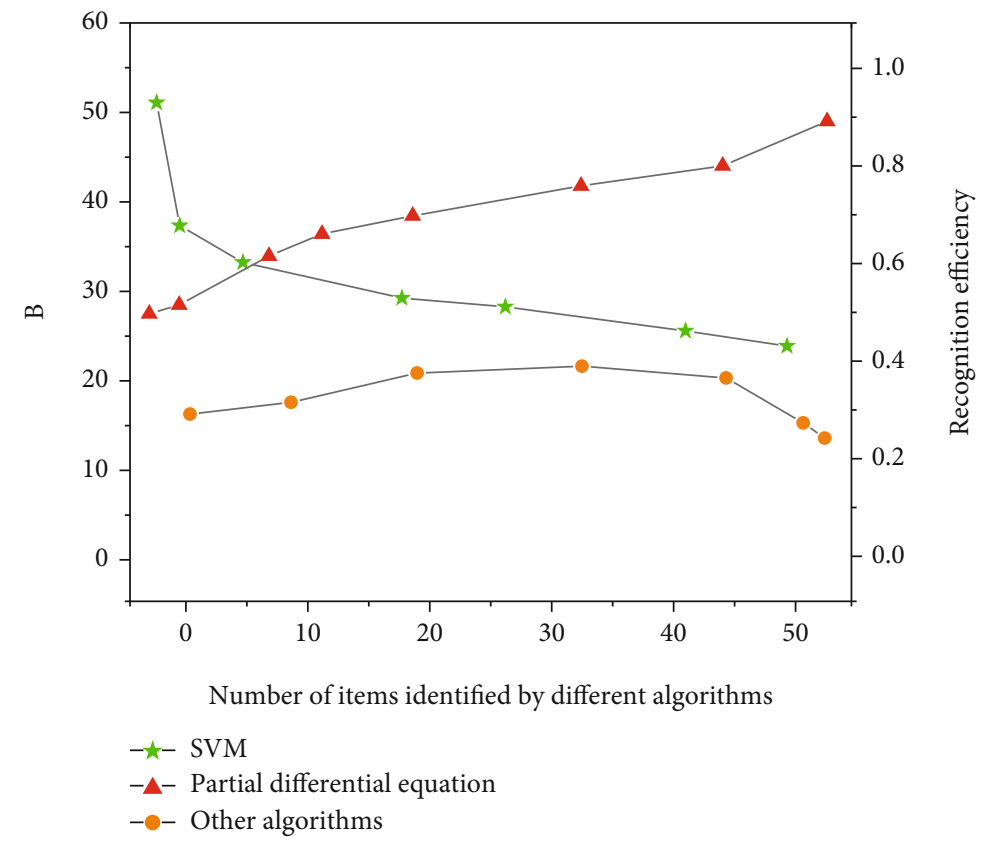

Figure 4: Average distance method and image recognition results based on LDA features.

$$
B=\lim _{x \longrightarrow \infty} \sum_{i=1}^{n} X_{i}^{2} \cdot \sum_{i=1}^{n}\left(X_{i}-\bar{X}\right)^{2}
$$

To solve the above problem, the Fisherface method is used to solve the "small sample" problem in image recognition by combining PCA and LDA. The basic idea of this method is to transform the features of the highdimensional space of the image sample to the low- dimensional features by using the PCA method of principal component analysis to ensure that the matrix obtained after projection is a nonsingular matrix. Then, the best discriminant vector is solved by the linear discriminant analysis (LDA) method. So, the final transformation matrix is obtained by combining the image features extracted from the above two image features [16].

By projecting the sample image to be tested onto the transformation matrix $W$, the effective features of the 


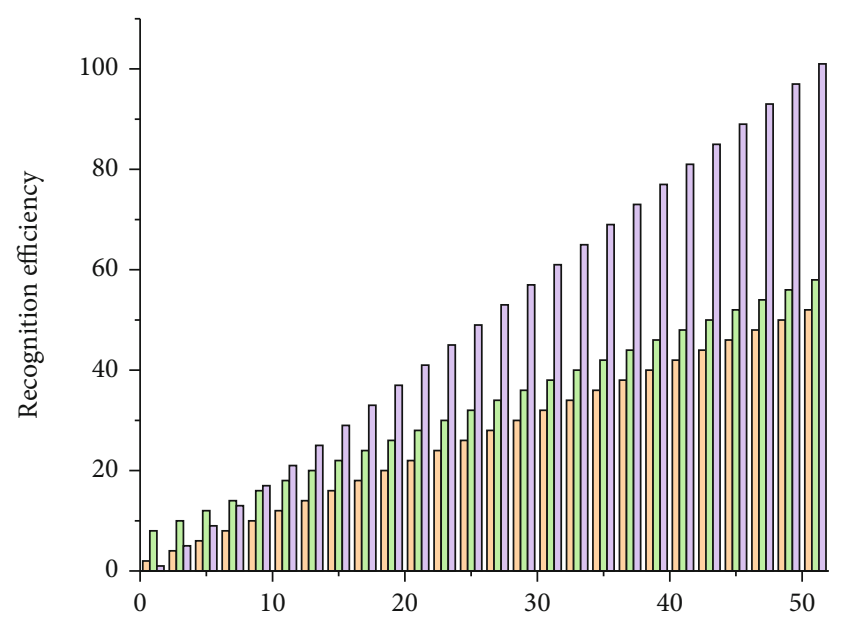

Number of identifications by different identification methods

Other methods

SVM

Partial Differential Equations

Figure 5: Comparison of experimental results.

product image can be obtained, and then, the obtained image features are fed to the classifier for recognition [31]. The following are the specific algorithmic steps for inventory image feature extraction using the LDA method.

(1) Suppose there are a total of c classes of commodity images to be recognized, and the total number of samples participating in training is $N$ belonging to these $c$ classes, with $W$ th class having the Nth sample. The prior probability that the training samples belong to the with a class of goods is $P(W, i)$, and the $M * N$-dimensional training commodity images are transformed into column vectors [17].

(2) Generate intraclass and interclass scatter matrices for the training sample set

(3) Using the PCA+LDA analysis method, a set of feature vectors with the best resolution is solved to form the transformation matrix. First, the reduced dimensional subspace WPCA of the overall scattering matrix of the training samples is obtained by the PCA method

\section{Experimental Procedure and Analysis}

The image recognition experiments conducted in this chapter are based on two feature extraction methods, PCA by principal component analysis and LDA by linear discriminant analysis, while to better utilize the color space features of the images for image recognition, the features of the three channels of the image $\mathrm{H}$ channel, S channel, and I channel are extracted separately in the HSI color space mentioned above. The commodity images are also recognized using the secondary classification combination method mentioned above. In addition, extensive experiments were also con- ducted on different classification methods such as the average distance method and error correction SVM alone to compare the correct recognition rates of various methods. By analyzing and comparing the experimental results, an image recognition method with better recognition results is identified for the recognition of supermarket shelf merchandise images in practical applications to verify the effectiveness of the quadratic classification image recognition algorithm in this paper [18].

4.1. Experimental Results of Capital Stock Charge Image Recognition Simulation. In this section, we will use simulation experiments of image recognition to test the effectiveness of image recognition methods based on primary classification method and secondary classification-based image recognition methods, respectively [19], record the relevant experimental results, analyze and compare the experimental results, and select the image recognition method with the best recognition effect as the method for image recognition of shelf goods.

To verify the effectiveness of the classification recognition method, firstly, a single classification method mean distance method and error correction SVM are combined with the principal component analysis method PCA and linear discriminant analysis LDA, respectively, and then, the images in the commodity library of this paper are recognized under different feature dimensions, and the following are the experimental results. The HSI color space features of commodity images are extracted using PCA, and then, the mean distance method and error correction SVM classifier are used to recognize the commodity images [20], respectively, and the experimental results are shown in Table 1.

To allow a more intuitive observation of the recognition results, to compare the variation of the recognition rate of each image recognition method with different feature dimensions, and for the comparison of the recognition results using different image feature extraction methods, a line graph of the variation of the correct recognition rate of shelf goods images with feature dimensions was drawn using MATLAB software as shown in Figure 3.

From the results in the figure, it can be seen that the overall recognition effect of the error correction SVM is more than one percentage point higher than the average distance method, and the recognition rate reaches a maximum of $87.65 \%$ because the error correction SVM classifier uses the error coding theory in communication technology and has a certain error correction capability. Therefore, the use of error correction SVM classifier in the recognition of commodity images achieves better results than the use of the average distance method, and its recognition effect is improved to some extent.

So that the change in recognition rate of commodity images can be observed more visually, a line graph of the change in recognition rate with the dimensionality of images for both image recognition methods was plotted using MATLAB software, as shown in Figure 4.

We performed a visual qualitative comparison of segmentation results between the method in this paper and unsupervised image segmentation algorithms such as 


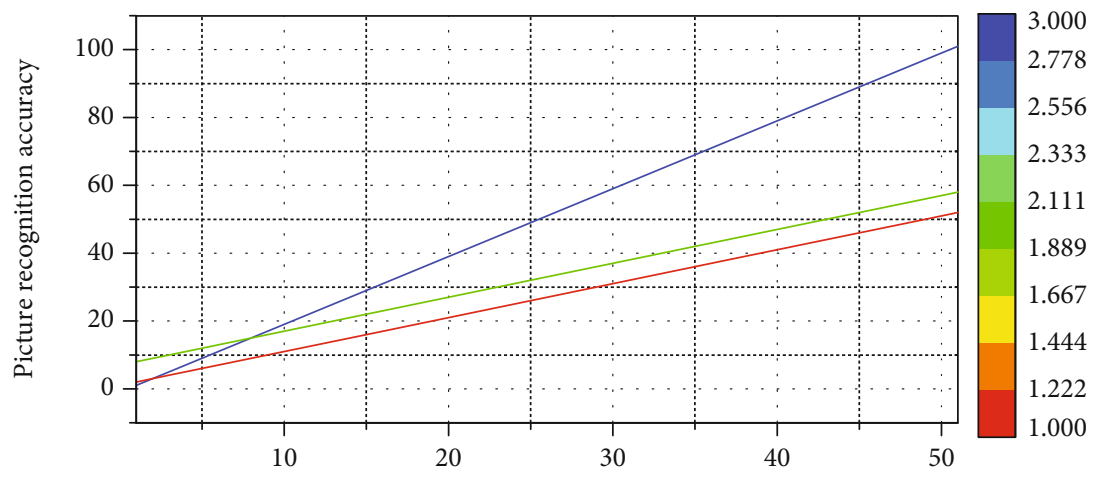

Number of image recognition between different methods

Figure 6: Accuracy of image recognition based on different algorithms.

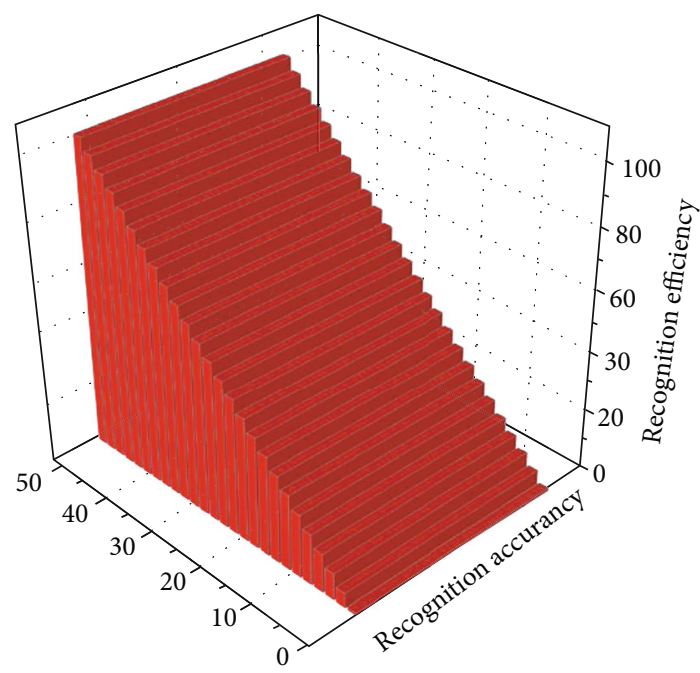

Image recognition based on partial differential equation

Figure 7: Comparison chart for different efficiencies.

GMM and Level-set, as shown in Figure 5. The spatial features used in this algorithm are extracted based on the superpixel algorithm, so we also compared the method in this paper with the latest superpixel segmentation algorithm, LSC, for the experiments.

4.2. Analysis of Experimental Results of Capital Stock Charge Image Recognition Simulation. Figure 6 shows the selected image to be segmented from the BSD500 dataset. Figure 6 shows the segmentation result of the GMM algorithm, the foreground image is segmented but due to the effect of the complex background image, and some regions such as clouds and grass are divided into foreground regions. The segmentation result of the Level-set algorithm is strongly influenced by the initial position; if the initial position is suitable, then the selection result is satisfactory.

In the subsequent experiments, plots of the coloring results of different methods for texture image I-V are shown. Compared to other methods, the model proposed in this chapter has a clear advantage. There is a color crossing for the literature method. For the literature's method, all the image colors have changed significantly. The literature's method is visible from the images where some areas are not colored. The literature's method also suffers from the difference in efficiency as in Figure 7, which is because the proposed model can preserve the contour lines of the grayscale image while preserving the colored edges.

\section{Conclusion}

In this paper, we study the image recognition method based on partial differential equations and the image coloring method based on variational differentiation. Image recognition is the most fundamental task in image processing, and noise largely blurs the detailed information of an image, bringing to the analysis and application of the image to be difficult. Partial differential equation-based image recognition methods can remove noise while protecting edges so that detailed information of the image is not corrupted; the method is of great interest to scholars. Image colorization, i.e., colorization of grayscale images, this technique can increase the image. The visual effect facilitates one to study and analyze the image in depth. The traditional methods of image coloring are color transfer-based shading coloring methods and color diffusion-based coloring methods. In recent years, with the improvement of computer performance, new deep learning-based coloring methods have also received extensive scholarly attention.

Firstly, we introduce the research background of image recognition and image coloring and their practical applications; then, we list the types of noise in images and introduce the current status of research on image recognition at home and abroad. As for image coloring, the common color spaces and the current research status of image coloring by domestic and foreign scholars are introduced. Section 2 introduces the related knowledge of image processing in detail. Firstly, several common partial differential equation-based image recognition models are briefly given, including the secondorder recognition model, the fourth-order recognition model, and the full-variance recognition model, and the image recognition problem is focused on. For the step effect that exists in the second-order partial differential equation when processing noise-laden images, the paper proposes 
two new fourth-order partial differential image recognition models and computes them numerically using the finite difference method, MATLAB. The simulation experiments illustrate that the proposed model can effectively remove multiplicative noise. In the experiments, the proposed first model uses a manual tuning trial thresholding approach, and the second model uses a histogram-based adaptive thresholding approach. Image recognition technology based on partial microequation can improve efficiency and speed in the future development direction. Development in this direction can always improve the effectiveness of its algorithm.

\section{Data Availability}

The data used to support the findings of this study are available from the corresponding author upon request.

\section{Conflicts of Interest}

The authors declare that they have no known competing financial interests or personal relationships that could have appeared to influence the work reported in this paper.

\section{Acknowledgments}

This work was supported by the Shaanxi Province Social Science Foundation Project: Research on the financing efficiency of enterprises on the New Third Board in Shaanxi Province, Project No. 2018D48.

\section{References}

[1] X. Jin, J. Che, and Y. Chen, "Weed identification using deep learning and image processing in vegetable plantation," IEEE Access, vol. 9, pp. 10940-10950, 2021.

[2] L. Chamoin, C. Jailin, M. Diaz, and L. Quesada, "Coupling between topology optimization and digital image correlation for the design of specimen dedicated to selected material parameters identification," International Journal of Solids and Structures, vol. 193-194, pp. 270-286, 2020.

[3] E. J. Shin and A. R. Koh, "Korean genderless fashion consumers' self-image and identification," Journal of the Korean Society of Clothing and Textiles, vol. 44, no. 3, pp. 400-412, 2020.

[4] L. Martín, J. Medina, and E. Upegui, "Assessment of imagetexture improvement applied to unmanned aerial vehicle imagery for the identification of biotic stress in espeletia. Case study: moorlands of Chingaza (Colombia)," Ciencia E Ingenieria Neogranadina, vol. 30, no. 1, pp. 27-44, 2020.

[5] D. H. Ko, S. H. Choi, J. M. Shin, P. Liu, and Y. H. Choi, "Structural image de-identification for privacy-preserving deep learning," IEEE Access, vol. 8, pp. 119848-119862, 2020.

[6] K. Niu, Y. Huang, W. Ouyang, and L. Wang, "Improving description-based person re-identification by multi-granularity image-text alignments," IEEE Transactions on Image Processing, vol. 29, pp. 5542-5556, 2020.

[7] H. He, J. Zheng, L. Liao, and Y. J. Chen, "Damage identification based on convolutional neural network and recurrence graph for beam bridge," Structural Health Monitoring, vol. 20, no. 4, pp. 1392-1408, 2021.
[8] N. Ahmed, H. M. Asif, G. Saleem, and M. U. Younus, "Image quality assessment for foliar disease identification (AgroPath)," Journal of Agricultural Research, vol. 59, no. 2, pp. 177-186, 2021.

[9] V. Krasheninnikov, O. Malenova, and A. Subbotin, "The identification of doubly stochastic circular image model," Procedia Computer Science, vol. 176, pp. 1839-1847, 2020.

[10] A. Ismail, "Auditee satisfaction impact on compliance and corporate image concerning Malaysian SMEs," Turkish Journal of Computer and Mathematics Education (TURCOMAT), vol. 12, no. 10, pp. 3436-3452, 2021.

[11] M. Matarazzo, L. Penco, G. Profumo, and R. Quaglia, “Digital transformation and customer value creation in made in Italy SMEs: a dynamic capabilities perspective," Journal of Business Research, vol. 123, pp. 642-656, 2021.

[12] A. Mueller and L. Sensini, "Determinants of financing decisions of SMEs: evidence from hotel industry," International Journal of Business and Management, vol. 16, no. 3, pp. 117127, 2021.

[13] C. Baah, D. Opoku-Agyeman, I. S. K. Acquah et al., "Examining the correlations between stakeholder pressures, green production practices, firm reputation, environmental and financial performance: evidence from manufacturing SMEs," Sustainable Production and Consumption, vol. 27, pp. 100114, 2021.

[14] S. Weaven, S. Quach, P. Thaichon, L. Frazer, K. Billot, and D. Grace, "Surviving an economic downturn: dynamic capabilities of SMEs," Journal of Business Research, vol. 128, pp. 109-123, 2021.

[15] H. Erlangga, "Effect of digital marketing and social media on purchase intention of Smes food products," Turkish Journal of Computer and Mathematics Education (TURCOMAT), vol. 12, no. 3, pp. 3672-3678, 2021.

[16] M. Hock-Doepgen, T. Clauss, S. Kraus, and C. F. Cheng, "Knowledge management capabilities and organizational risk-taking for business model innovation in SMEs," Journal of Business Research, vol. 130, pp. 683-697, 2021.

[17] C. P. K. Coffie, Z. Hongjiang, I. A. Mensah, R. Kiconco, and A. E. O. Simon, "Determinants of FinTech payment services diffusion by SMEs in sub-Saharan Africa: evidence from Ghana," Information Technology for Development, vol. 27, no. 3, pp. 539-560, 2021.

[18] X. Y. Chen, M. S. Zhang, S. Jiang et al., "An SMES-based current-fed transformerless series voltage restorer for DCload protection," IEEE Transactions on Power Electronics, vol. 36, no. 9, pp. 9698-9703, 2021.

[19] T. Alvarez, L. Sensini, C. Bello, and M. Vazquez, "Management accounting practices and performance of SMEs in the hotel industry: evidence from an emerging economy," International Journal of Business and Social Science, vol. 12, no. 2, pp. 24-35, 2021.

[20] S. Magrizos, E. Apospori, M. Carrigan, and R. Jones, "Is CSR the panacea for SMEs? A study of socially responsible SMEs during economic crisis," European Management Journal, vol. 39, no. 2, pp. 291-303, 2021. 